\title{
Legibility of Displayed Place Names by In-Vehicle Navigation Devices
}

\author{
Kazumoto MORITA , Jin'ichi MASHIKO, Shin'ichiro ITOH and Takeo OKADA
}

Traffic Safety and Nuisance Research Institute

6-38-1 Shinkawa Mitaka-city Tokyo 181

Received December 4, 1995

\begin{abstract}
The authors have conducted mainly indoor experiments in which they tested the legibility of three place names in Tokyo area displayed by a navigation device by having the observers read the place names as quickly and accurately as possible. The three place names simulate a road sign at an intersection. The reading time and the reading accuracy of the three place names are examined under various experimental conditions, such as the size of the displayed place name, the position of the display device, the observer's age and other factors. For example, to reproduce experimental conditions, three kinds of display devices and display positions are tested; first, head-up display in the central vision, second, head-up display in the peripheral (near central) vision, and third, the conventional $L C D$ display in the peripheral vision. There are thirty observers in three age ranges (in their 20s, 40s, and 60s), with ten in each group. The authors have revealed how the experimental conditions affect the legibility of the three place names displayed by in-vehicle navigation devices.
\end{abstract}

KEYWORDS: automobile, safety, navigation system, legibility, head-up display

\section{Introduction}

Navigation systems that provide route guidance by presenting a simple map on a display device can now be found in many cars. It is necessary that the display is as easy to read as possible to prevent the driver from taking his attention away from the road.

Various factors such as display position and display contents are related to the amount of time needed to read the display (reading time). For example, since head-up displays (HUDs), which project the display information on the windshield, reduce the movement of the driver's line of sight, they can be expected to require less time to read than traditional dashboard displays. Concerning the contents of the display, the extent of the detail of the map provided to the driver can be expected to influence reading time. Further, the matter of legibility does not only depend on the display device itself, but also on the driver himself; one can predict that the age of the driver influences reading time. Although there have been studies on navigation systems ${ }^{121334)}$, many points still remain unclear.

In this study, we have examined the legibility of the three place names displayed by in-vehicle navigation devices which simulate a road sign at an intersection. The display devices including the HUD, the display positions, the size of the place names, the ages of the observers, and other factors were examined as variables in mainly indoor experiments to see how they affected reading time and the accuracy in which the place names were read.

\section{Indoor Experimental Method}

The indoor experiments were conducted in a dark room in which the lights on the ceiling were turned off. Figure 1 shows the overall experimental design.

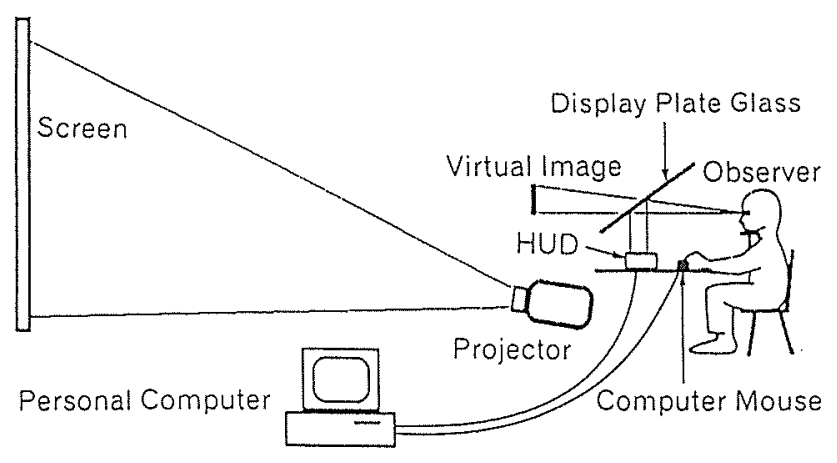

Fig. 1 Diagram of Experiment Design 
Before the experiment, we filmed the road in front of a moving car from the interior of that car. In the indoor experiments, the filmed road scenery was projected onto a white screen that was located approximately $5.6 \mathrm{~m}$ in front of the observer to simulate the conditions of a moving car. Although the luminance of the road scenery projected onto the screen differed depended on the measured object, according to measurement results through the display plate glass, luminance of road and intersection scenery was $3-5$ $\mathrm{cd} / \mathrm{m}^{2}$, that of the rear part of a whitish vehicle was $3-7$ $\mathrm{cd} / \mathrm{m}^{2}$, and that of the rear portion of a blackish vehicle was $1-4 \mathrm{~cd} / \mathrm{m}^{2}$.

\subsection{Display Devices}

We used the two types of display devices in the experiments which were a HUD and a conventional LCD dashboard display.

The HUD had a built-in LCD screen through which transmitted light was emitted. This light was reflected by a mirror in the device and appeared as a virtual image on a display plate glass that was mounted in front of the observer. Because the surface of the display plate glass was coated by oxidized titanium, it had a reflectance of $20 \%$. It was mounted at an incline of 31 degrees from horizontal, and the transmissivity of the plate glass from the observation direction was $72 \%$.

The conventional LCD dashboard display was a 5.6-inch liquid crystal TV screen.

\subsection{Display Positions}

The direction when the observer looked straight ahead was defined as the observer's central vision. There were two types of display positions for the HUD. One type of display position was in the central vision as shown in Figure 2. The other type, as shown in Figure 3, was 7.0 degrees left of and 2.3 degrees below central vision. That is, it was in the peripheral vision (near the central vision). In the case of the HUD in the central vision, the distance between the observers eyes and the virtual image was $1.65 \mathrm{~m}$. Both the HUD in the central vision and HUD in the peripheral vision were observed as they overlapped the projected road scenery.

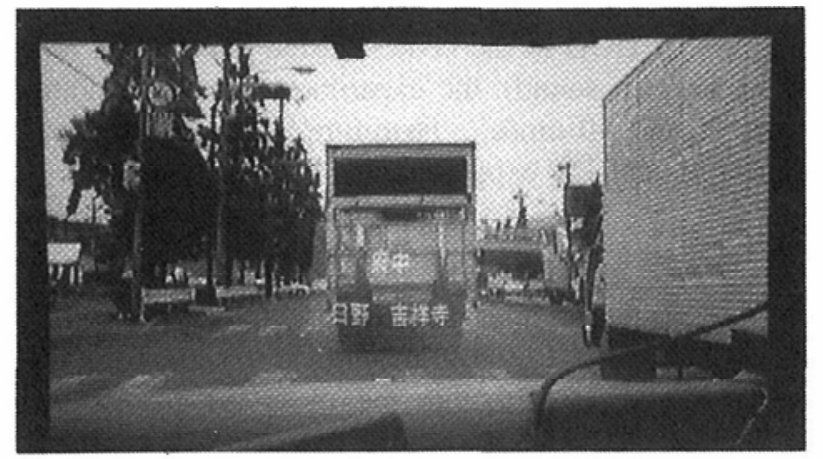

Fig. 2 Example of HUD in the Central Vision

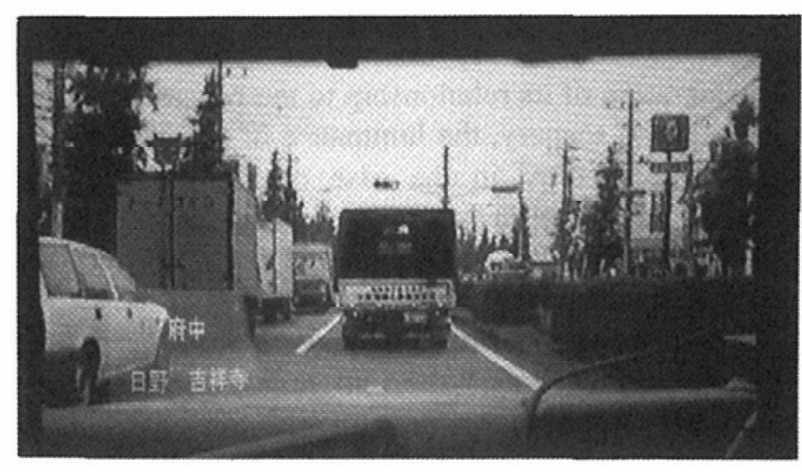

Fig. 3 Example of HUD in the Peripheral Vision

The position of the conventional LCD display followed that of standard navigation devices on the dashboard. It was in a peripheral position that was 31 degrees to the left of and 24 degrees below central vision (standard Japanese cars have their steering wheel on the right side of the vehicle). In this case, the LCD did not overlap the road scenery in front and was observed in a lower left direction. The straight-line distance from the observer's eyes to the surface of the LCD display was $0.84 \mathrm{~m}$.

\subsection{Displayed Place Names}

In these experiments, we have adopted place names which simulate a road sign at an intersection. Thirty place names from the Tokyo area were selected and three names at a time were used for each display. The reason three names were shown is that an ordinary intersection road sign will probably present the destinations that are straight ahead, to the left, and to the right. An example of a place name display is shown in Figure 4.

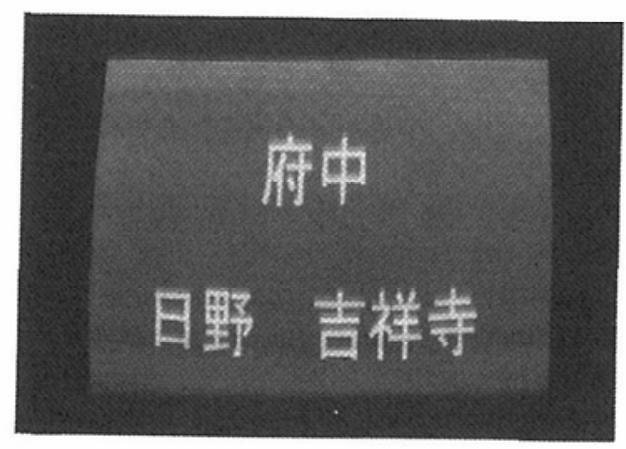

Fig. 4 Example of a Place Name Display

The size of place names on a HUD can be set to the four kinds of sizes: visual angles in vertical direction of one character are 0.57 degrees, 0.70 degrees, 0.83 degrees, and 1.02 degrees. The overall size of the displayed image is three lines in the vertical direction and 6 characters in the horizontal direction.

The visual angle in the vertical direction of one character is used as a base thereafter and each size is called " 0.57 degree size," etc. The color of all place name displays was 
green (with chromaticity coordinates of $\mathrm{x}=0.277$ and $\mathrm{y}=$ $0.593)$.

In consideration of its relationship to the brightness of the background road scenery, the luminance of the display was approximately $70 \mathrm{~cd} / \mathrm{m}^{2}$ in the case of the HUD. This value was obtained by measuring the luminance of only the displayed image in a darkened room and did not include the effects of the road scenery brightness.

As for the conventional LCD display, in accordance with preparatory experiments, the brightness was set at approximately $40 \mathrm{~cd} / \mathrm{m}^{2}$ to prevent the observers from feeling that it was too bright. We used the only size that equaled the 0.83 degree HUD image size. The height of one character on the LCD screen was approximately $12 \mathrm{~mm}$. The color of the displayed names was also green (with chromaticity coordinates of $\mathrm{x}=0.274$ and $\mathrm{y}=0.598$ ).

We used a personal computer for producing display images, controlling experimental conditions, analyzing data, etc. The display images were created in advance and kept on the computer's hard disk. For each experiment, a particular display image was selected randomly and sent to the HUD or conventional LCD display device.

\subsection{Experimental Procedure}

The following procedures were repeated for the experiment.

- The observer looks at the road scenery on the screen with no place names being displayed on it.

- The experimenter displays three place names by pressing the computer keys without warning.

- The observer reads the displayed names and presses the button on the computer mouse when he is finished.

- The display disappears as soon as the button is pressed and the reading time is automatically recorded in the computer.

- The observer orally tells the three names to the experimenter.

The time from when the display was made to when the observer pressed the mouse button was measured. Consequently, strictly speaking, reading time actually includes the time needed to read the display and the time needed to press the mouse.

The observer was instructed to "read the displayed place names as quickly and accurately as possible." Consequently, both reading time and reading accuracy are treated as analytical items.

\subsection{Other Experimental Conditions}

In addition to the three display positions (HUD in the central vision and the HUD in the peripheral vision, and LCD display in the peripheral vision), we also included the experimental conditions described below.

In actuality, the driver may drive with his attention diffused as he listens to the radio or stereo. Consequently, we carried out experiments to see how measurement results would be affected if the observer listened to news on the radio. Radio news was recorded on a cassette tape which was played for the observer.

Besides displaying place names without warning to the observer, the names were also displayed after initiating a warning sound (a beeping sound) to see how it would affect the experimental results. The sound was made 1.0 second before the display went on and lasted for 0.3 seconds. The sound was followed by an 0.7 second blank before the display went on. In this case, such as in the case of an LCD display in the peripheral vision, the observer was told that he could look in the direction of the display at the same time he heard that warning sound.

The experiments were conducted in the order of the experimental conditions given below.

(1) HUD in the central vision without the radio news or warning sound

(2) LCD display in the peripheral vision without the radio news or warning sound

(3) HUD in the central vision while listening to the radio

(4) LCD display in the peripheral vision while listening to the radio

(5) HUD in the central vision with warning sound

(6) LCD display in the peripheral vision with warning sound

(7) HUD in the peripheral vision without the radio news or warning sound

(8) HUD in the peripheral vision while listening to the radio

(9) HUD in the peripheral vision with warning sound

For condition (1) (HUD in the central vision without the radio news or warning sound), the four types of display sizes given previously were used as variables in the experiments. For the rest of the conditions [(2) - (9)], only one display size $(0.83$ degree size $)$ was used. Consequently, when comparing and analyzing the experimental results of (1) and those of (2) through (9), only the 0.83 degree size from among the four display sizes in condition (1) was aggregated in congruence with each other.

The experiments were conducted 10 times each for each condition. Since four display sizes were used for condition (1) (HUD in the central vision without the radio news or warning sound), the experiment for this condition was carried out 40 times. Therefore, each observer went through a total of 120 experiments.

\subsection{Observers}

The observers set their head position by placing their chins on a chin rest $1.00 \mathrm{~m}$ in height and observed the road scenery. However, although the observers placed their chins on the chin rest, they were not made to constantly stare straight ahead and were free to look at the entire road scenery. 
There was a total of 30 observers (16 males and 14 females): 10 in their 20 s (average age $=24.7$ ), 10 in their 40 s (average age $=44.2$ ), and 10 in their 60 s (average age $=$ 64.0).

It is inevitable that the observers' getting used to the experiment or their fatigue influences reading time as the experiment progresses. Therefore, besides displaying the place names randomly, the group was halved so that 15 were experimented on in the order of (1) to (9) as given earlier and the other 15 were experimented in the order of (9) to (1). This was done to exclude the effects of accustomization and fatigue from the aggregate data on reading time and accuracy.

\subsection{Tests on Degree of Familiarity with Place Names}

It is believed that displayed place names may either be familiar or unfamiliar to the observers. We carried out tests to see if such familiarity or unfamiliarity would cause any differences in reading time. For this purpose, the following five-level scheme was used to evaluate the familiarity of each place name before the experiments for each observer.

1. Very unfamiliar

2. Slightly unfamiliar

3. Neither

4. Slightly familiar

5. Very familiar

\section{Indoor Experiment Results}

The two items measured in the experiments are reading time and reading accuracy. By checking the rate of correct answers given by all observers for all place names, it is shown that the observers have read the place names accurately since the rate is an extremely high at $99.3 \%$. Consequently, the focus here will be on reading time and we will not carrying out analysis on reading accuracy any further.

In order to statistically study differences in reading time due to varying conditions, a normal distribution test ${ }^{5 /}$ was carried out to determine if the hypothesis that "the averages of two groups are equal" could be rejected or not. A normal distribution test is a testing method in which the value of $\mathrm{z}$ (below) is equal to standard normal distribution $\mathrm{N}(0,1)$ based on the hypothesis that there is no difference between the average of two groups .

$$
\begin{aligned}
& \mathrm{z}=\frac{\bar{x}_{1}-\bar{x}_{2}}{\sqrt{\frac{\sigma_{1}^{2}}{n_{1}}+\frac{\sigma_{2}^{2}}{n_{2}}}} \\
& \text { Here: } \\
& \bar{x}_{\mathrm{i}} ; \mathrm{i}=1,2: \text { sample average of each group } \\
& \sigma_{\mathrm{i}} ; \mathrm{i}=1,2: \text { standard deviation of each group } \\
& n_{\mathrm{i}} ; \mathrm{i}=1,2: \text { sample number of each group }
\end{aligned}
$$

The results of our study concerning the hypothesis could be rejected or not with a significant level of $1 \%$ are described below.

\subsection{Difference in Reading Time Due to Age}

We have analyzed the HUD in the central vision data in the case when there is not radio news or warning sound in order to examine difference in reading time due to age (Figure 5). There is not much difference in reading time between observers in their 20 s (average: 1.59 seconds) and 40s (average: 1.69 seconds). However, the average reading time of those in their $60 \mathrm{~s}$ is much longer at 2.17 seconds. It is also shown that standard deviation increases with age.

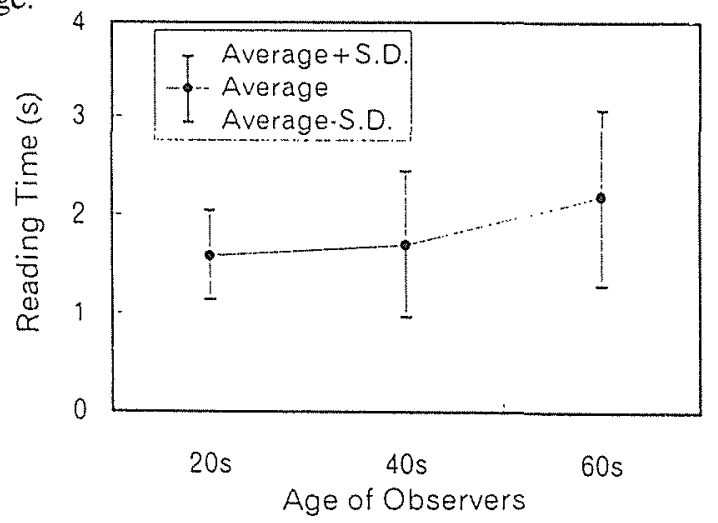

Fig. 5 Reading Time Differences Due to Age

By conducting a statistical test on averages, we have determined that there are reading time differences with significant levels of $1 \%$ when comparing the 60 s group with the $40 \mathrm{~s}$ group and the $60 \mathrm{~s}$ group with the $20 \mathrm{~s}$ group.

\subsection{The Effects of Display Size}

We have examined the differences in reading time in connection with the four types of display sizes based on the HUD in central vision data in the case when there is not radio news or warning sound (Figure 6). There is a tendency for reading time to be longer with the 0.57 degree size. In cases where the display size is 0.70 degrees or larger, there are no remarkable differences in reading time.

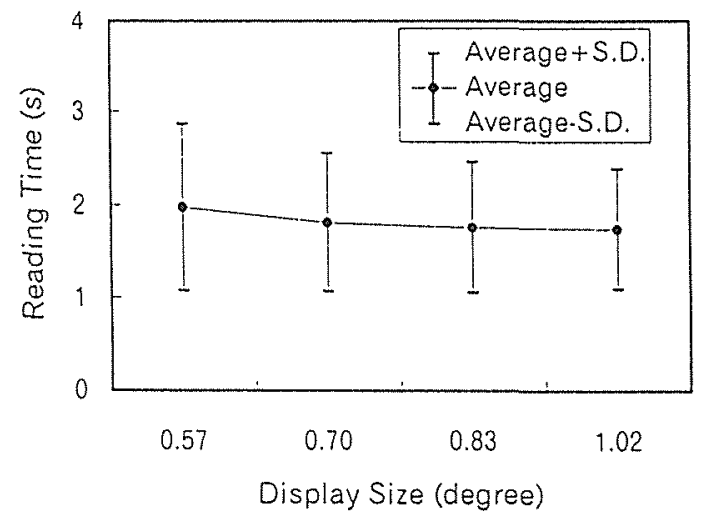

Fig. 6 Reading Time Differences Due to Display Size 
A statistical test on averages reveals that there is a difference in reading time with a significant level of $1 \%$ when comparing the 0.57 degree size with the 0.83 degree size and the 0.57 degree size with the 1.02 degree size.

By looking at size in relation to age (Figure 7), we can see that reading time is longer for the 0.57 degree size in the case of the $60 \mathrm{~s}$ group. A statistical test on averages for this group shows that there is a reading time difference with a significant level of $1 \%$ when comparing the 0.57 degree size with the 1.02 degree size. The difference in reading time due to display size is not observed with a significant level of $1 \%$ for the $20 \mathrm{~s}$ and $40 \mathrm{~s}$ groups.

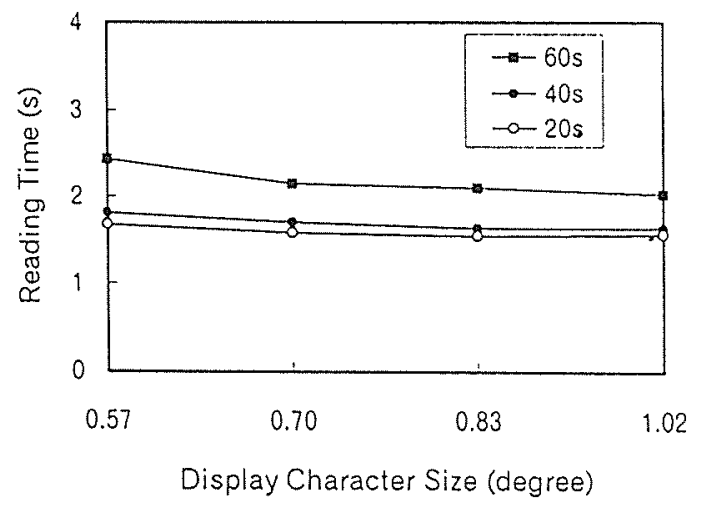

Fig. 7 Effect of Display Size and Age

Consequently, it is shown that it is necessary to use a certain display size for older persons.

\subsection{The Effects of Display Position}

We have analyzed the effects of display position in the case when there is not radio news or warning sound. As shown in Figure 8, there is little difference in reading time between the case of the HUD in the central vision (average: 1.76 seconds) and the case of the HUD in the peripheral vision (average: 1.85 seconds). Reading time, however, is longer in the case of the LCD display in the peripheral vision (average: 2.18 seconds). With the LCD display in the peripheral vision, it is necessary for the observer to notice the display and move his line of vision. Compared with the HUDs, approximately 0.4 seconds more time is needed to read the display.

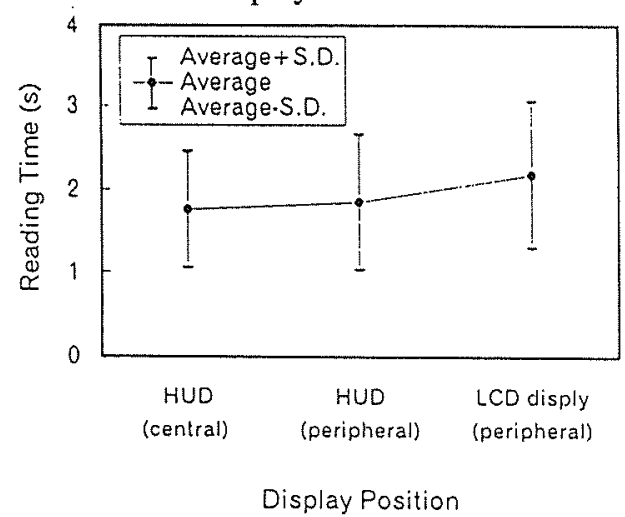

Fig. 8 Reading Time Differences Due to Display Position
A statistical test on averages shows that there is a reading time difference with a significant level of $1 \%$ when comparing the LCD display in the peripheral vision with the HUD in the central vision and the LCD display in the peripheral vision with the HUD in the peripheral vision. The difference in reading time between the HUD in the central vision and the HUD in the peripheral vision is not observed with a significant level of $1 \%$.

\subsection{Reading Time When Listening to the Radio}

Figure 9 shows the reading time when the observers listen to the radio. The reading times when listening to the radio for the HUD in the central vision (average: 1.78), the HUD in the peripheral vision (average 1.89) and the LCD display in the peripheral vision (average: 2.17 ) show little if any change when compared to Figure 8 . The differences in reading time due to radio listening are not determined with a significant level of $1 \%$ by a statistical test.

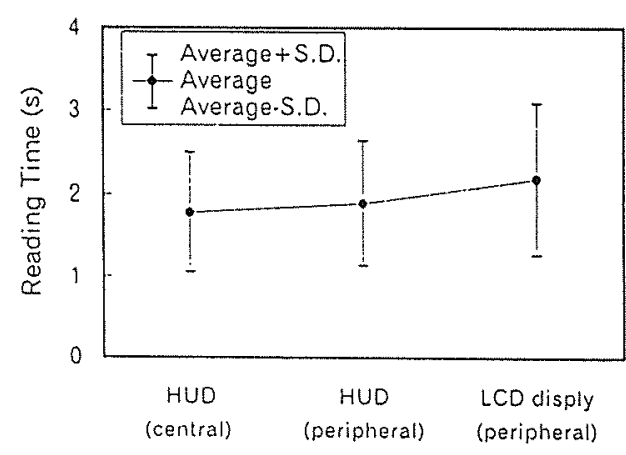

Display Position

Fig. 9 Reading Time When Listening to the Radio

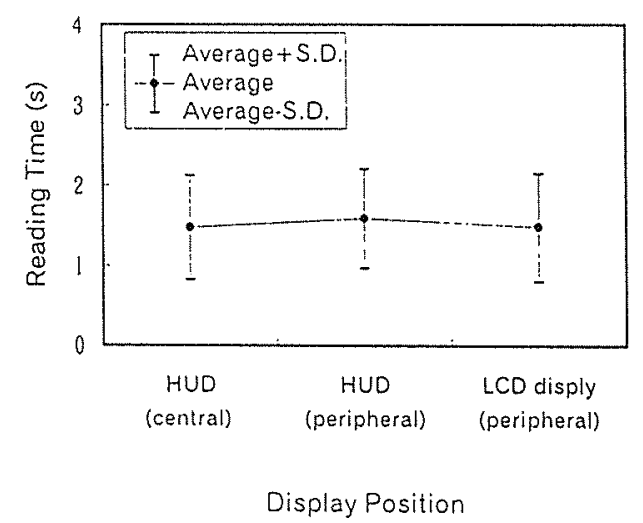

Fig. 10 Reading Time When There is a Warning Sound

\subsection{Reading Time with Warning Sound}

Figure 10 shows the reading time when there is a warning sound one second before the display. No differences are recognized among three different display 
positions. The reading time even in the case of the LCD display in the peripheral vision (average: 1.48 seconds) is shortened to about the same length as that of the HUD in the central vision. The statistical test results do not show a difference with a significant level of $1 \%$, confirming that there is a tendency for reading time average values to be almost the same regardless of the type of display device and position when there is a warning sound.

Further, in the case of the HUD in the central vision, reading time is 1.47 seconds when there is a warning sound, which is 0.28 seconds less than when there is no warning sound. In the case of the HUD in the peripheral vision, reading time is 0.27 seconds less when there is a warning sound. Meanwhile, the reading time for the LCD display in the peripheral vision is 0.70 seconds less when there is a warning sound. This tendency for reading time to be less for all three types of display positions when there is a warning sound is confirmed by a statistical test with a significant level of $1 \%$.

In the case of the LCD display in the peripheral vision, the reading time when there is a warning sound is believed shorter because the sound reduces the time needed for the observer to notice the display and move his field of vision. Further, there actually is a reduction in reading time with a warning sound in the case of the HUD in the central vision as well. That suggests that the difference in reading time is related to the degree of concentration on the display when there is a warning sound.

Figure 11 (for the LCD display in the peripheral vision with a warning sound) shows the relationship of reading time to age. As in the case when there is no warning sound, reading time increases with age. In the case of Figure 11, a statistical test on reading time averages shows that there is a reading time difference with a significant level of $1 \%$ when observers in their 60 s are compared with observers in their $40 \mathrm{~s}$ and when observers in their $60 \mathrm{~s}$ are compared with observers in their $20 \mathrm{~s}$.

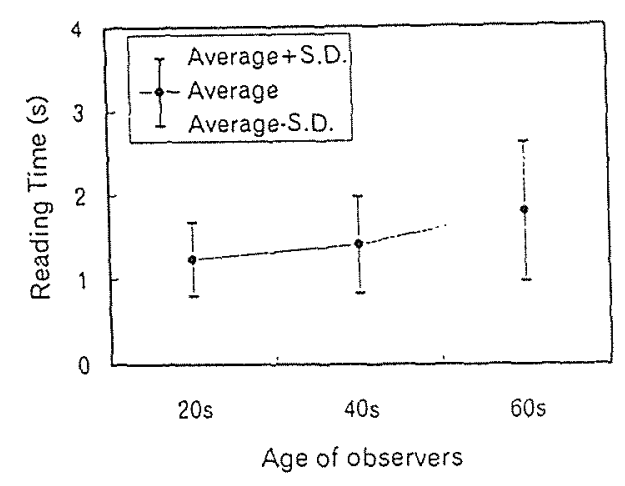

Fig. 11 Reading Time Differences Due to Age When There is a Warning Sound

(In the case of a LCD display in the peripheral vision)

\subsection{The Effects of Degree of Familiarity with Place Name}

In these experiments, the observers were made to read three place names that were shown to them. It is predicted that reading time would be less when the place names are familiar to the observers. Consequently, the relationship between degree of familiarity and reading time has been examined based on the results of a questionnaire administered to each observer. In the questionnaire, points are assigned to answers based on the degree of familiarity. For example, 1 point is given for a name that is "very unfamiliar" and 2 points are given for one that is "slightly unfamiliar." Thus, five points are given for a name that is "very familiar." Since three names are displayed at one time in the experiments, an average number of points for those three names is taken as a representative value (hereinafter referred to as "average degree of familiarity"). Naturally, the average degree of familiarity of a display with the same three names varies among different observers. The minimum average degree of familiarity of 1.0 results when three "very unfamiliar" names are displayed and the maximum average degree of familiarity of 5.0 results when three "very familiar" names are displayed.

For the analysis, the HUD in the central vision data in the case when there is not radio news or warning sound is used. From that data, only the data of the 15 observers on whom the experiments were conducted in forward order is used. This is because it is believed that the observers would become familiar with even unfamiliar place names after being exposed to them repeatedly as in the case when the experiments were conducted in the reverse order.

Figure 12 shows the relationship between the average degree of familiarity of three place names and reading time. Although there are no remarkable differences in the overall data, it can be seen that reading time is greater when the average degree of familiarity is greater or equal to 1 and less than 2 .

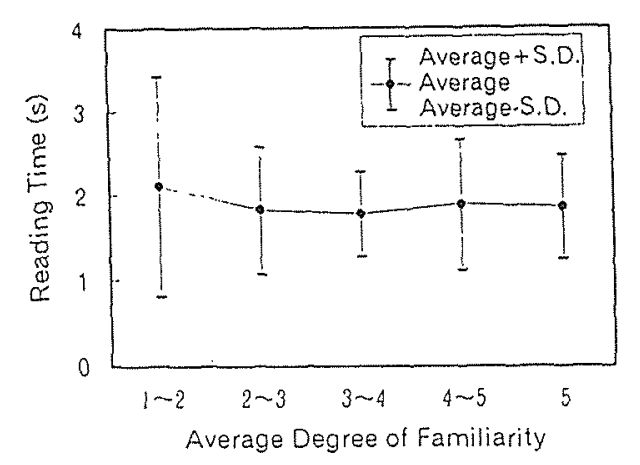

Fig. 12 Reading Time Differences and Average Degree of Familiarity

(Here, $1-2,2-3$, etc. expresses greater or equal to 1 and less than 2, greater or equal to 2 and less than 3 , etc.) 
A statistical test shows that there is a difference in reading time with a significant level of $5 \%$ when the range of average degree of familiarity of 1 to 2 is compared with that of 3 to 4 . With other ranges, statistical average differences with a significant level of $5 \%$ are not observed.

Based on the above, we can see that reading time tends to be slightly longer when the place names are unfamiliar to the observer. In these experiments, since place names from the Tokyo area were selected and used on observers who were from the Tokyo area, it is conceivable that there were almost no cases where the names were seen for the first time even though the observers may have answered that they were "very unfamiliar." We may presume that reading time would be greater if the observers were in an area for the first time and were shown names that they had never seen before.

\section{Legibility of Place Names in Driving Experiments}

Driving experiments using a passenger car on a test course were carried out in addition to indoor experiments to study the legibility of place names while driving.

\subsection{Procedure of Driving Experiments}

The basic experimental methods were the same as those of the indoor experiments. The same conventional LCD display used in the indoor experiments was mounted 31 degrees left and 18 degrees below the observer's eyes (Figure 13). The straight-line distance from the observer's eyes to the surface of the LCD display was approximately $0.76 \mathrm{~m}$.



Fig. 13 LCD Display in Driving Experiments

After practice runs on the course, the test was conducted by repeating the following procedures.

- The observer drives the experimental vehicle at $60 \mathrm{~km} / \mathrm{h}$ in a straight line. At this time, the observer holds a computer mouse in one hand.

- The experimenter, who rides together with the observer, displays three place names by pressing the computer keys.

- The observer begins to read the place names that are displayed and presses the mouse button when he is finished.

- The display disappears as soon as the button is pressed and the reading time is recorded automatically in the computer.
- The observer stops the experimental vehicle and tells the experimenter the place names he has read.

To simulate actual traffic conditions and to induce a mental load on the observer, he was also made to drive 20 to $25 \mathrm{~m}$ behind a lead car that traveled at $60 \mathrm{~km} / \mathrm{h}$.

There were 15 observers ( 7 in their 20 s, 3 in their 30 s, 4 in their $40 \mathrm{~s}$, and 1 in his $50 \mathrm{~s}$; average age $=33.5$ ).

Experiments were conducted for cases without a warning sound and with a warning sound. Ten experiments for each case were conducted on each observer. Therefore, there were 150 experiments on cases without a warning sound and 150 experiments on cases with a warning sound.

\subsection{Driving Experiment Results}

Concerning reading accuracy, the overall rate of correct answers for all observers is very high at $99.7 \%$. As in the indoor experiments, the observers have accurately read the place names.

The relationship between reading time and cases with and without a warning sound is shown in Figure 14. The average reading time is 1.95 seconds when there is no warning sound and 1.48 when there is a warning sound. Consequently, a difference in reading time due to the presence or absence of a warning sound is recognized. A statistical test on the average shows a difference with a significant level of $1 \%$ due to the presence or absence of a warning sound.

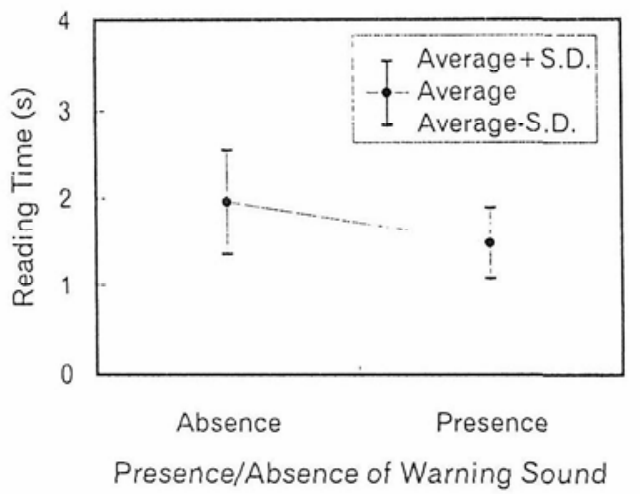

Fig. 14 Reading Time Differences Due to Presence/Absence of Warning Sound During Driving Experiments

The average reading time for LCD display in the peripheral vision obtained in the indoor experiments is compared with that obtained in the driving experiment. When there is no warning sound, the reading time is 2.18 seconds in the indoor experiment and 1.95 in the driving experiment, that is, almost no difference is observed. When there is a warning sound, the reading time values are the same at 1.48 seconds in both the indoor and driving experiments. Although the experimental results cannot be precisely compared because of differences in the age composition of the observers and the position of the LCD 
display device, the reading time for both the indoor and driving experiments are quite similar.

\section{Conclusion}

We have carried out mainly indoor experiments concerning the legibility of displays by automobile navigation devices. The displayed information is three place names that would likely appear in route guidance at an intersection. Three types of display devices and positions are used: HUD in the central vision, HUD in the peripheral vision, and conventional LCD display in the peripheral vision. We obtained the following information concerning reading time and reading accuracy.

(1) The observers read the place names accurately.

(2) There is a tendency for older observers to have a longer reading time than young and middle-aged observers. Further, it is necessary to make the display at a certain size or larger for older observers.

(3) Reading time is shorter for HUDs than for the LCD display in the peripheral vision. A warning sound increases the observer's concentration and shortens reading time even in the case of the HUDs.

(4) There is no significant difference in reading time even when the radio is on.

(5) Slight decreases in reading time is observed when the displayed place names are familiar to the observer. We may assume that reading time may be longer if place names that the observer does not know and have never seen before are displayed.

(6) The reading time results of driving experiments concerning the presence or absence of a warning sound with a conventional a LCD display in the peripheral vision are similar to those of the indoor experiments.

\section{References}

(1) Thomas A. Dingus, et al.: "Attentional Demand Requirements of an Automobile Moving-Map Navigation System," Transpn. Res.-A, Vol.23, No.4, pp.301-315 (1989)

(2) Jonathan Walker, et al.: "In-Vehicle Navigation Devices: Effects on the Safety of Driver Performance," Proc. of Vehicle Navigation and Information Systems Conference, part 1 (1991)

(3) S. Shekhar, et al.: "Design and Validation of Headup Displays for Navigation in IVHS," Proc. of Vehicle Navigation and Information Systems Conference, part 1 (1991)

(4) Jun'ichi Fukano, et al:: "Automotive Head-Up Displays for Navigation Use," Proc. of the 14th International Technical Conference on Enhanced Safety of Vehicles, paper No. 94-S2-O-02 (1994)

(5) Takuro Kishine: "Statistics Theory from Beginning to Application," Yokendo, pp.207-216 (1976) 